\title{
Motion-Corrected, Super-Resolution Reconstruction for High-Resolution 3D Cardiac Cine MRI
}

\author{
Freddy Odille ${ }^{1,2,3}$, Aurélien Bustin ${ }^{4,5}$, Bailiang Chen ${ }^{1,2,3}$, \\ Pierre-André Vuissoz ${ }^{1,2}$, and Jacques Felblinger ${ }^{1,2,3}$ \\ ${ }^{1}$ U947, Inserm, Nancy, France \\ ${ }^{2}$ IADI, Université de Lorraine, Nancy, France \\ ${ }^{3}$ CIC-IT 1433, Inserm, Nancy, France \\ ${ }^{4}$ Computer Science, Technische Universitat Munchen, Munich, Germany \\ ${ }^{5}$ GE Global Research Center, Garching, Germany
}

\begin{abstract}
Cardiac cine MRI with 3D isotropic resolution is challenging as it requires efficient data acquisition and motion management. It is proposed to use a 2D balanced SSFP (steady-state free precession) sequence rather than its 3D version as it provides better contrast between blood and tissue. In order to obtain 3D isotropic images, 2D multi-slice datasets are acquired in different orientations (short axis, horizontal long axis and vertical long axis) while the patient is breathing freely. Image reconstruction is performed in two steps: (i) a motioncompensated reconstruction of each image stack corrects for nonrigid cardiac and respiratory motion; (ii) a super-resolution (SR) algorithm combines the three motion-corrected volumes (with low resolution in the slice direction) into a single volume with isotropic resolution. The SR reconstruction was implemented with two regularization schemes including a conventional one (Tikhonov) and a feature-preserving one (Beltrami). The method was validated in 8 volunteers and 10 patients with breathing difficulties. Image sharpness, as assessed by intensity profiles and by objective metrics based on the structure tensor, was improved with both SR techniques. The Beltrami constraint provided efficient denoising without altering the effective resolution.
\end{abstract}

Keywords: Magnetic resonance imaging, super-resolution, motion-compensated reconstruction.

\section{Introduction}

High-resolution, 3D isotropic cine imaging of the heart is challenging because it requires lengthy acquisitions, even with efficient imaging sequences, and therefore advanced patient motion management. Such an imaging technique would be useful for understanding complex anatomy and function in congenital heart diseases or for imaging small cardiac structures such as the atrium or the valves. It might also help reducing the variability of ventricular volumetric assessment in cardiovascular diseases (stroke volume, ejection fraction...) which is generally high with the conventional 2D cine image stacks due to the difficulty of segmenting the myocardium near the base of the ventricles. 
The b-SSFP (balanced steady-state free precession) sequence is one of the most widely used imaging techniques for cardiac cine imaging. This is because it provides the highest signal-to-noise ratio per unit time among all known sequence, with good $\mathrm{T}_{2} / \mathrm{T}_{1}$ contrast [1]. Moreover when the slice thickness is not too small (5 to $10 \mathrm{~mm}$ ) the $2 \mathrm{D}$ b-SSFP does not suffer from severe motion-induced signal dropouts thanks to its fully balanced gradients. Unlike its 3D version, the 2D b-SSFP provides excellent contrast between tissues and blood/vessels due to the inflow effect (fresh spins moving in/out of the slice) [1]. A multi-slice 2D b-SSFP acquisition is therefore a good candidate but it suffers from poor resolution in the slice direction. One possible way to overcome this limitation is to combine multiple 2D stacks acquired in different orientations using a super-resolution (SR) reconstruction [2-4]. Applying SR techniques to chest or abdominal imaging is difficult because the image stacks to be combined need to be perfectly aligned. Rigid motion correction techniques have been combined with SR algorithms [5] but nonrigid motion is even more challenging and has not been addressed yet.

In this paper we propose a method for high-resolution, 3D isotropic cine imaging of the heart. The acquisition strategy consists of multiple 2D image stacks with different orientations obtained by a 2D multi-slice b-SSFP sequence during free breathing. The image reconstruction consists of two steps: (i) a motion-compensated $3 \mathrm{D}$ cine reconstruction of each stack; (ii) a super-resolution reconstruction combining the multiple cine volume stacks into an isotropic cine volume. The objective of this paper is to show the feasibility of the method in actual volunteer and patient experiments.

\section{Image Reconstruction}

\subsection{Motion-Compensated 3D Cine Reconstructions}

In the first step each stack of $2 \mathrm{D}$ slices (from one particular volume orientation) is reconstructed with a nonrigid motion compensation approach named cine-GRICS (generalized reconstruction by inversion of coupled systems) [6, 7]. Here the method reconstructs $N_{p h}=32$ volumes corresponding to 32 phases of the cardiac cycle. In order to reconstruct a given cardiac phase, k-space data within a cardiac acceptance window (here $W_{\text {card }}=120 \mathrm{~ms}$ ) are selected. As described hereafter the motion-compensated reconstruction for this cardiac phase is formulated as the joint reconstruction of a 3D image $\rho_{i}(i=1,2,3$ for SA, HLA, VLA) and a motion model $\alpha$ accounting for respiratory motion and for cardiac motion within the acceptance window.

The motion model approximates 3D time-varying nonrigid displacement fields $u(x, y, z, t)$ in the imaged volume such that $u(x, y, z, t) \simeq \sum_{k=1}^{N_{s}} \alpha_{k}(x, y, z) s_{k}(t)$ as described in Ref. [7], with some motion signals $s_{k}(t)$ acquired simultaneously with the k-space data $m$. Here we use $N_{s}=5$ motion signals: $\left[s_{1}(t) s_{2}(t)\right]$ are the signals from two pneumatic belts (chest and abdomen); $\left[s_{3}(t) s_{4}(t)\right]$ are their first-order time derivatives; and $s_{5}(t)$ is the cardiac phase signal derived from the ECG recordings $\left(s_{5}(t)\right.$ has values between 0 and the mean cardiac cycle length in $\left.\mathrm{ms}\right)$. 
For each cardiac phase the joint reconstruction is achieved by solving:

$$
\min _{\left(\rho_{i}, \alpha\right)}\left\|E(\alpha, s) \rho_{i}-m\right\|^{2}+\lambda\left\|\rho_{i}\right\|^{2}+\mu\|\nabla \alpha\|^{2} .
$$

Here $E(\alpha, s)$ denotes the forward acquisition model, i.e. the motion-corrupted 2D multi-slice acquisition. It includes a 3D nonrigid transformation operator for each motion state, followed by a slice selection, receiver coil sensitivity weightings, 2D Fourier transforms and k-space sampling operators similar to the description in Ref. [7]. The respiratory signals $s_{1}(t) \ldots s_{4}(t)$ are centered on their most likely value (i.e. end-expiratory plateaus) so that all image stacks are reconstructed in a consistent motion state. Problem (1) is solved by a multi-resolution Gauss-Newton scheme, alternating between optimization with respect to $\rho$ and $\alpha$ as described in [7].

\subsection{Super-Resolution Reconstruction}

In a second step the $N$ motion-compensated 3D cine volumes (obtained from different orientations, $N=3$ in this study) are combined using SR reconstruction. For a given cardiac phase if $\rho_{1} \ldots \rho_{N}$ denote the $N$ motion-compensated 3D images, the SR image is found by solving:

$$
\min _{x} \sum_{i=1}^{N}\left\|D_{i} B_{i} T_{i} x-\rho_{i}\right\|^{2}+\lambda R(x) .
$$

Here $T_{i}$ is a rigid image transformation that takes the SR image from the desired reconstructed orientation to the orientation of the $i^{\text {th }}$ acquisition (i.e. it is an interpolation operator that can describe an arbitrary orientation); $D_{i} B_{i}$ is a slice selection operator including a blurring operator $B_{i}$ (i.e. a sum in the slice direction in the range of the slice thickness) and a downsampling operator $D_{i}$ (in the slice direction).

Two regularizers were tested in this study: Tikhonov regularization, i.e. $R(x)=$ $\|x\|^{2}$ and Beltrami regularization [8], i.e. $R(x)=\left(1+\beta^{2}|\nabla x|^{2}\right)^{1 / 2}$. The Beltrami regularizer is a modified version of the well-known total variation and has better feature-preserving properties. In the case of Tikhonov regularization the SR reconstruction is a linear least-squares problem and is solved by a conjugate gradient solver. For the Beltrami regularization a primal-dual projected gradient scheme is used [8].

\section{MRI Experiments}

8 healthy volunteers and 10 patients with major breathing difficulties (Duchenne muscular dystrophy) underwent a cardiac functional MRI. Experiments were conducted on either a $1.5 \mathrm{~T}$ Signa HDxt (10 patients and 5 volunteers) or a $3 \mathrm{~T}$ Signa HDxt scanner (3 volunteers) (General Electric Healthcare, USA). For both the volunteers and the patients written informed consent was obtained and both studies were approved by an ethical committee. A 2D multi-slice b-SSFP sequence was used to cover the left ventricle in three different orientations: short-axis (SA), horizontal longaxis (HLA) and vertical long axis (VLA). The orientations were those planned by the 
MR technologist (they were chosen to be independent but not strictly orthogonal). All acquisitions were performed during free breathing and were ungated (retrospective gating was included in the cine-GRICS reconstruction step). Typical parameters were: $224 \times 224$ acquisition matrix, 20 frames (i.e. $20 \mathrm{k}$-spaces acquired per slice), native inplane resolution $1.6 \times 1.6 \mathrm{~mm}^{2}, 8 \mathrm{~mm}$ slice thickness, TE/TR=1.8/4.1ms.

The cine-GRICS motion correction step provided three cine volumes (with 32 cardiac phases) of resolution $1.6 \times 1.6 \times 8 \mathrm{~mm}^{3}$ (by solving Eq. (1)) which were combined by the SR reconstruction to form an isotropic volume of resolution $1.6 \mathrm{x} 1.6 \mathrm{x}$ $1.6 \mathrm{~mm}^{3}$ (by solving Eq. (2)). The SR reconstruction was performed on the smallest cube encompassing the intersection volume of the three image stacks.

\section{$4 \quad$ Validation}

In order to validate the method we compared the SR reconstructed images (with Tikhonov and Beltrami regularization) against the native images. We first analyzed the images visually with cine-loop movies. We also drew intensity profiles across the ventricle to highlight differences in effective resolution in various oblique directions.

We then proposed a quantitative assessment. Isotropic images are expected to provide good depiction of features/edges in all three dimensions unlike the images with native resolution which are blurred in the slice direction. It is proposed to assess the presence of features in specific directions using the structure tensor [9]. The structure tensor $S$ of a 3D image $I$ characterizes, for each voxel $p$ in the image, the local orientation of the anatomical structure based on the gradients in a small neighborhood of $p$ :

$$
S[p]=\left[\begin{array}{lll}
S_{x x}[p] & S_{x y}[p] & S_{x z}[p] \\
S_{x y}[p] & S_{y y}[p] & S_{y z}[p] \\
S_{x z}[p] & S_{y z}[p] & S_{z z}[p]
\end{array}\right],
$$

with $S_{i j}[p]=(W *(\partial I / \partial i \times \partial I / \partial j))[p]$ for $(i, j) \in\{x, y, z\}^{2}$.

Here $W$ is a Gaussian convolution kernel defining the size of the neighborhood. Before computing the structure tensor, the SA images (native resolution of $1.6 \times 1.6 \mathrm{x}$ $8 \mathrm{~mm}^{3}$ ) were interpolated to the same isotropic resolution as the SR images (i.e. $1.6 \mathrm{x}$ $1.6 \times 1.6 \mathrm{~mm}^{3}$ ) using a windowed sinc interpolation (Lanczos window).

We compared the following metrics based on the structure tensor in the different reconstructions: $\left\langle S_{x x}>,\left\langle S_{y y}>\right.\right.$ and $\left\langle S_{z z}>\right.$ (i.e. the average of $S_{x x}, S_{y y}$ and $S_{z z}$ respectively over the reconstructed volume). $\left\langle S_{x x}\right\rangle$ and $\left\langle S_{y y}\right\rangle$ can be viewed as the amount of details (edges, features...) present in the SA plane while $<S_{z z}>$ relates to the direction orthogonal to SA. It is expected that the SR images can preserve directional information from the native in-plane resolution and that they can retrieve additional information in the orthogonal direction $(z)$ from the VLA and HLA images (increased $<S_{z z}>$ ). Statistical differences between the native and SR images were assessed by Wilcoxon signed rank tests. A significance level of 5\% was used. 


\section{$5 \quad$ Results}

The time needed to acquire the three cine stacks was approximately $10 \mathrm{~min}$ (4 $\mathrm{min}$ for SA, 3 min for HLA and 3 min for VLA). The cine-GRICS reconstruction time was $180 \mathrm{~min}$ (SA+HLA+VLA) using a parallel C++ code running on a cluster of 16 workstations; the SR step for a full cine dataset took 14/16 min (Tikhonov/Beltrami) using Matlab (single-thread, sequential reconstruction of the 32 cardiac phases).

Example reconstructed images are shown in Fig. 1. Images are shown both in enddiastole and in end-systole position. The visual comparison between the native SA images and the SR images shows preserved image quality in the short-axis plane with a slight noise amplification for the Tikhonov regularization. Important differences are observed in the through-plane direction, i.e. in the VLA and HLA orientations. Several anatomical structures and vessels were not visible in the native SA images but were recovered by both SR reconstruction techniques. Differences are mostly noticed near the base and near the apex of the left ventricle where the structures are orthogonal to the slice direction (see arrows in Fig. 1).

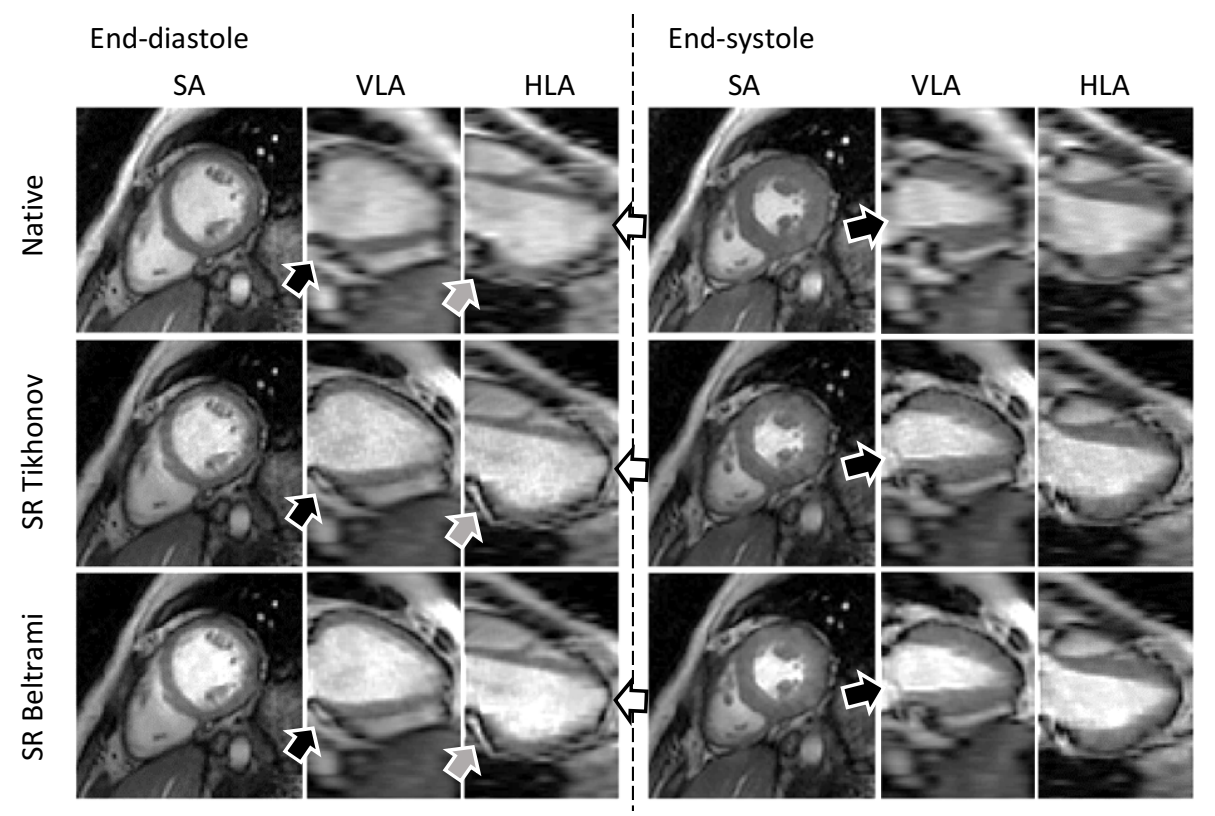

Fig. 1. Example 3D cine datasets from a patient: native short-axis images (top row), superresolution with Tikhonov (middle row) and with Beltrami regularization (bottom row). The arrows point out noticeable anatomical details which were not visible in the native images but were recovered by both SR techniques, especially near the base and apex of the left ventricle.

Example intensity profiles drawn across the left ventricle are shown in Fig. 2. Again the resolution improvement compared to the native images can be observed, especially near the mitral plane and at the apex. Structures like the myocardial wall and papillary muscles appear much sharper in SR images. Beltrami regularized images appear less noisy than Tikhonov images. 


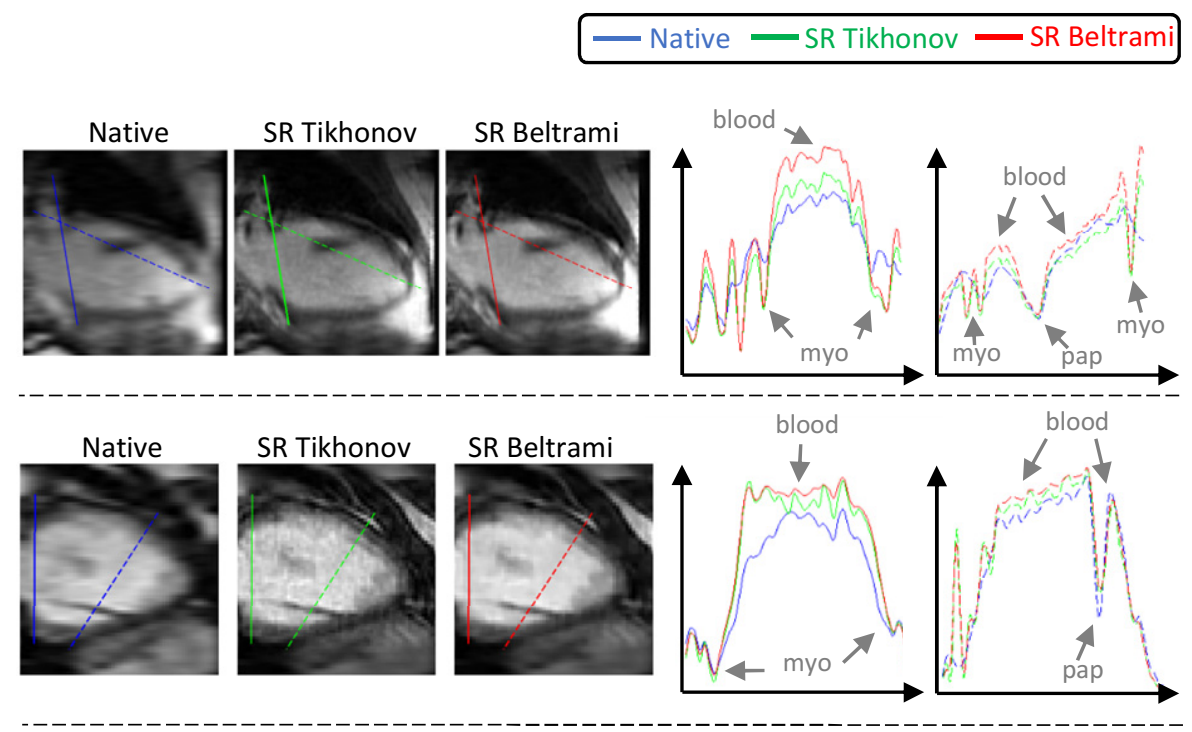

myo = myocardial wall; pap = papillary muscle

Fig. 2. Example intensity profiles from two volunteers (top and bottom row). Two line profiles were drawn on the images (left side: native and SR images) with various oblique orientations and the corresponding intensity profiles are shown on the right. Finer details better observed in the SR images and Beltrami regularized images appear less noisy than Tikhonov ones.
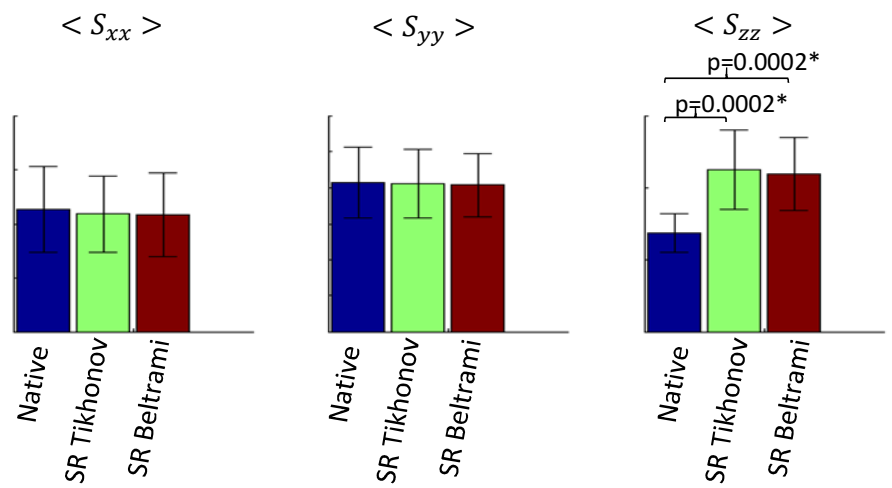

Fig. 3. Directional information (derived from the structure tensor $S$ ) contained in the native short-axis images and in the SR images (with Tikhonov and Beltrami regularization) in the short-axis plane $\left(<S_{x x}>\right.$ and $\left\langle S_{y y}\right\rangle$ ) and in the orthogonal direction $\left.\left(<S_{z z}\right\rangle\right)$. Differences between native and SR images were not significant for $\mathrm{x}$ and $\mathrm{y}$ directions and were significant for $\mathrm{z}$ direction.

Measures of directional information, as measured by the structure tensor metrics in the 18 subjects (volunteers and patients), are summarized in Fig 3. In-plane information (SA plane) was preserved between native SA images and SR images, as differences in 
$<S_{x x}>(\mathrm{p}>0.1)$ and $\left\langle S_{y y}>(\mathrm{p}>0.6)\right.$ were not statistically significant. Information in the orthogonal direction was improved with SR reconstruction compared to the native images since $\left\langle S_{z z}\right\rangle$ was significantly different $(\mathrm{p}=0.0002)$. Beltrami denoising did not seem to affect this resolution enhancement as differences in all three metrics between Tikhonov and Beltrami regularized images were not significant $(\mathrm{p}>0.5)$.

\section{Discussion and Conclusion}

The proposed reconstruction combines nonrigid motion-correction and super-resolution. Our hypothesis was that SR reconstruction can be guaranteed if two conditions are met: (i) images with good quality and good motion-consistency can be provided as inputs of the SR algorithm, i.e. the input volumes need to have high in-plane resolution, minimal blurring/ghosting artifacts, and the misalignments from one volume to another should be minimal; (ii) an efficient SR solver can be implemented, i.e. efficient regularization schemes can be implemented in order to solve this under-determined inverse problem. For these reasons the joint reconstruction of image and motion (GRICS approach) and the feature-preserving Beltrami regularization were key technical choices. Our results in healthy volunteers and patients with breathing difficulties show the feasibility of the technique.

Alternative methods for SR cine reconstruction include dictionary learning approaches $[10,11]$ which do not explicitly require motion correction. Our cine-GRICS approach has the advantage of using a motion model that inherently compensates intra-image motion (ghosting/blurring), intra-stack motion (slice-to-slice consistency) and inter-stack motion (stack-to-stack consistency). Compared to other total variation schemes [12] our Beltrami scheme yields similar results with faster convergence [8].

The proposed approach still has limitations that should be mentioned. Despite its high efficiency the $b$-SSFP sequence is sensitive to $B_{0}$ field inhomogeneity that may result in dark banding artifacts. Although the requirement in terms of $\mathrm{B}_{0}$ homogeneity is less for the 2D b-SSFP than it is for its 3D version, it does rely on efficient shimming in the volume to be scanned. It should also be noted that both the in-flow effect and the location of the dark-band artifacts depend on the slice orientation and may result in outliers in the native images. This might be overcome with robust SR algorithms [13]. Another limitation is that the motion correction and the SR steps were applied sequentially. The final reconstruction might still be improved by merging the two steps into a single optimization problem. Such an approach would consist of searching for the isotropic SR image directly from the motion-corrupted k-space data.

In conclusion we have proposed a strategy for 3D isotropic cine MRI. The data acquisition consists of multiple stacks of 2D b-SSFP datasets with different orientations. Efficient SR reconstruction of an isotropic 3D cine dataset was rendered possible by (i) advanced nonrigid motion correction (using 3D cine GRICS) providing artifactfree and motion consistent images for each stack; (ii) regularized inversion of the SR model (with either Tikhonov or feature-preserving Beltrami regularization). The feasibility has been demonstrated in healthy volunteers and patients with breathing difficulties. The approach might be adapted to other imaging sequences or applications such as 3D late gadolinium enhancement. 
Acknowledgement. The authors would like to thank AFM (French association against myopathy) for funding the patient study (ID C13-04). This publication was supported by the European Commission, through Grant Number 605162. The content is solely the responsibility of the authors and does not necessarily represent the official views of the EU.

\section{References}

1. Scheffler, K., Lehnhardt, S.: Principles and applications of balanced SSFP techniques. European Radiology 13(11), 2409-2418 (2003)

2. Greenspan, H., Oz, G., Kiryati, N., Peled, S.: MRI inter-slice reconstruction using superresolution. Magnetic Resonance in Medicine 20(5), 2409-2418 (2003)

3. Gholipour, A., Estroff, J., Warfield, S.: Robust super-resolution volume reconstruction from slice acquisitions: application to fetal brain MRI. IEEE Transactions on Medical Imaging 29(10), 1739-1758 (2010)

4. Plenge, E., Poot, D., Bernsen, M., Kotek, G., Houston, G., Wielopolski, P., van der Weerd, L., Niessen, W., Meijering, E.: Super-resolution methods in MRI: Can they improve the trade-off between resolution, signal-to-noise ratio, and acquisition time? Magnetic Resonance in Medicine 68(6), 1522-2594 (2012)

5. Rousseau, F., Glenn, O., Iordanova, B., Rodriguez-Carranza, C., Vigneron, D., Barkovich, J., Studholme, C.: Registration-based approach for reconstruction of high-resolution in utero fetal MR brain images. Academic Radiology 13(9), 1072-1081 (2006)

6. Vuissoz, P., Odille, F., Fernandez, B., Lohezic, M., Benhadid, A., Mandry, D., Felblinger, J.: Free-breathing imaging of the heart using $2 \mathrm{D}$ cine-GRICS (generalized reconstruction by inversion of coupled systems) with assessment of ventricular volumes and function. Journal of Magnetic Resonance Imaging 35(2), 340-351 (2012)

7. Odille, F., Vuissoz, P., Marie, P., Felblinger, J.: Generalized reconstruction by inversion of coupled systems (GRICS) applied to free-breathing MRI. Magnetic Resonance in Medicine 60(1), 146-157 (2008)

8. Zosso, D., Bustin, A.: A primal-dual projected gradient algorithm for efficient Beltrami regularization. UCLA CAM Report, 14-52 (2014)

9. Bigun, J., Granlund, G., Wiklund, J.: Multidimensional orientation estimation with applications to texture analysis and optical-flow. IEEE Trans. Pattern Anal. Mach. Intell. 13(8), 775-790 (1991)

10. Caballero, J., Price, A., Rueckert, D., Hajnal, J.: Dictionary learning and time sparsity for dynamic MR data reconstruction. IEEE Trans. Med. Imaging 33(4), 979-994 (2014)

11. Bhatia, K., Price, A., Shi, W., Hajnal, J., Rueckert, D.: Super-resolution reconstruction of cardiac MRI using coupled dictionary learning. In: Proc. of ISBI, pp. 947-950 (2014)

12. Tourbier, S., Bresson, X., Hagmann, P., Thiran, J.-P., Meuli, R., Cuadra, M.B.: Efficient total variation algorithm for fetal brain MRI reconstruction. In: Golland, P., Hata, N., Barillot, C., Hornegger, J., Howe, R. (eds.) MICCAI 2014, Part II. LNCS, vol. 8674, pp. 252-259. Springer, Heidelberg (2014)

13. Kuklisova-Murgasova, M., Quaghebeur, G., Rutherford, M., Hajnal, J., Schnabel, J.: Reconstruction of fetal brain MRI with intensity matching and complete outlier removal. Med. Image Anal. 16(8), 1550-1564 (2012) 